\title{
Mir-608 Overexpression in Idiopathic Pulmonary Fibrosis (IPF) is Related to Acetylcholinesterase Single Nucleotide Polymorphism (SNP)
}

Gali Epstein Shochet ( $\sim$ gali.epstein@clalit.org.il )

Meir Medical Center https://orcid.org/0000-0002-3417-9171

\section{Lilach Israeli-Shani}

Meir Medical Center

Isabelle Kains

Tel Aviv University Sackler Faculty of Medicine

Ori Wand

Meir Medical Center

David Shitrit

Meir Medical Center

\section{Research}

Keywords: microRNA, IPF, ILD, SNP, CdC42

Posted Date: July 28th, 2020

DOl: https://doi.org/10.21203/rs.3.rs-48065/v1

License: (9) This work is licensed under a Creative Commons Attribution 4.0 International License. Read Full License 


\section{Abstract}

Background: Idiopathic pulmonary fibrosis (IPF) is a chronic progressive disease that causes scarring of the lungs. The disease is associated with the Usual Interstitial Pneumonia (UIP) pattern, which was not yet fully recapitulated by an animal model. Therefore, the disease is considered 'human specific'. miRNA608 is a primate specific miRNA with many potential targets, such CdC42 and Interlukin-6 (IL-6) that were previously implicated in IPF pathology.

Objective: To test miR-608 expression and its targets in IPF patient samples.

Methods: RNA was extracted from Formalin fixed paraffin embedded (FFPE) tissue sections ( $\mathrm{N}=18)$. miRNA-608 expression and Cdc42 and IL- 6 levels were analyzed by qPCR. Acetylcholinesterase (AChE) is another target of miRNA-608. Its' rs17228616 allele has a single-nucleotide polymorphism (SNP) causing weakened miR-608 interaction (C2098A). Thus, DNA was extracted from whole blood samples from 56 subjects with fibrosing interstitial lung disease (ILD) and this region was sequenced for assessment of rs17228616 allele polymorphism.

Results: MiR-608 is significantly overexpressed in IPF samples, in comparison with controls $(p<0.05)$. Cdc42 and IL- 6 levels were lower in the IPF patient samples compared with control samples $(p<0.001$ and $p<0.05$, respectively).

The frequency of the rs 17228616 minor A-allele was 17/56 (30.4\%) with all patients being heterozygous. This result is significant vs. the published Israeli cohort of healthy individuals, which reported $17 \%$ prevalence of this allele in healthy control volunteers $(p=0.01, \mathrm{OR}=2.1, \mathrm{Cl} 95 \%$ [1.19-3.9]).

Conclusion: MiR-608 is overexpressed in IPF patients. While the exact mechanism remains to be discovered, it could potentially promote fibrotic disease.

\section{Introduction}

Idiopathic pulmonary fibrosis (IPF) is a chronic disease that causes scarring of the lungs. The disease is characterized by progressive worsening of dyspnoea and decline in lung function. Many patients with IPF have sub-clinical or clear co-morbid conditions including pulmonary hypertension, gastroesophageal reflux, obstructive sleep apnoea, obesity, emphysema, as well as depression and anxiety [1, 2].

The disease is limited to the lungs, and is associated with the histopathologic and/or radiologic pattern of Usual Interstitial Pneumonia (UIP). So far, no animal model fully recapitulated the UIP pattern [3] and the disease is considered 'human specific'.

miRNAs are a non-coding RNA sequences, of about 22 nucleotides long that have complementary sequences to particular regions of the mRNA, often in the $3^{\prime} U T R$, through which regulation occurs. Regulation generally proceeds via either mRNA degradation or inhibition, leading to gene silencing, with 
the degree of inhibition highly depending on the degree of complementarity between the miRNA and its corresponding mRNA target.

Single-nucleotide polymorphisms (SNPs) are strongly associated with susceptibility to various diseases, including IPF [4-6]. SNPs within miRNA sequences may therefore change properties of the resulting inhibition by altering degree of complementarity $[7,8]$.

miRNA-608 (hsa-mir-608) is a long (25 nucleotides) primate specific miRNA. It has many potential targets, such as Rho GTPase CdC42 and Interlukin-6 (IL-6), bearing much experimental evidence $[9,10]$. Both IL-6 and CdC42 have been shown to be involved in both anxiety and IPF [11]. Therefore, we tested miR-608 expression and its targets in IPF patient samples.

\section{Materials And Methods}

\section{RNA purification and quantitative PCR (qPCR):}

Total RNA (including miRNA) was extracted from Formalin fixed paraffin embedded (FFPE) tissue sections taken from 18 IPF patients and 8 Control samples, using the miRNeasy FFPE kit (Qaigen, USA) according to the manufacturer's instructions.

mRNA was converted to cDNA using the reverse transcription kit (Applied Biosystems, UK). Reactions were performed with SYBR Green PCR master mix (Applied Biosystems, UK). Primer sequences (purchased from Hylabs, Israel) are listed in Table 1. 18S served as the reference housekeeping gene. Primers were normalized by specific cDNA standard curves obtained from known amounts of cDNA.

Table 1

List of primers

\begin{tabular}{|lll|}
\hline & Forward (5'-3') & Reverse (5'-3') \\
\hline $\begin{array}{l}\text { CDC42 variant } 1 \\
(\text { NM_001791) }\end{array}$ & CTGTCAAGTATGTGTGGAGTGTTCTG & CTCTTCTTCGGTTCTGGAGGCT \\
$\begin{array}{l}\text { CDC42 variant 2 } \\
(\text { NM_044472) }\end{array}$ & TGCACTTACACAGAAAGGCC & CTTCTTCGGTTCTGGAGGCT \\
18S & AGGAATTGACGGAAGGGCAC & GGACATCTAAGGGCATCACA \\
\hline IL-6 & GGTACATCCTCGACGGCATCT & GTGCCTCTTTGCTGCTTTCAC \\
\hline
\end{tabular}

miRNA was converted into cDNA using the qScript microRNA cDNA synthesis kit (Quanta Biosciences, USA) and was then amplified using Perfecta Universal PCR primer kit (Quanta Bioscience, USA). qPCR was performed with Perfecta SYBR Green using specific primers for mir-has-608 (Quanta bioscience, USA). The reference genes (Snord 44 and RNV6) primers were supplied with the kit (sequence not available). 


\section{Human Blood Samples:}

Whole blood samples were collected from 56 subjects with fibrosing interstitial lung disease (ILD). DNA was purified from these samples using QaiSymphony (Qaigen). Their demographic data, as well as disease progression and final diagnosis were also recorded.

\section{Gene sequencing:}

DNA was amplified using PCRBIO HS Taq master mix (PCR Biosystems) with specific primers for the AchE: Forward 5'-CGCTGGAGCTCCTACATGGT-3' and Reverse 5'-ATAGACTCGGCCCCGTGAT-3'. Products were purified using FastAP Thermosensitive Alkaline Phosphatase (Thermo-Fisher scientific). Then, sequencing was performed using BigDye ${ }^{\mathrm{TM}}$ Terminator v3.1 Cycle Sequencing Kit (Thermo-Fisher Scientific) according to manufacturer's instructions. The target sequence was analysed by 3130 Genetic analyser (Applied Biosystems) for determining A/C allele single nucleotide polymorphism (SNP) at rs17228616.

\section{Statistical Analysis:}

Statistical analysis was done using GraphPad Prism version 7.00 for Windows (GraphPad Software, La Jolla California USA, www.graphpad.com). ANOVA was performed to compare differences between multiple cohorts. Paired Student's t-tests were employed to analyze differences between the two groups. An effect was considered significant when the P-value was $<0.05$. All experiments were repeated at least three times.

\section{Ethical approval:}

This study was approved by the local Ethics Committee (MMC-18-18). Informed consent was obtained from all subjects.

\section{Results}

\section{MiR-608 is overexpressed in lung tissue samples from IPF patients}

The expression of miR-608 in was evaluated in IPF FFPE patient samples. Interestingly, a significant overexpression of miR-608 was found in IPF patient samples, in comparison with controls $(p<0.05$, Figure 1A). Using the miRDB search (http://mirdb.org/cgi-bin/search.cgi), 975 possible targets for miR-608 were identified. These results were then subjected to WEB-based Gene set analysis toolkit (WebGestalt, http://www.webgestalt.org). We analysed the overrepresentation analysis (ORA) for disease (OMIM) functional database. Interestingly, IPF was the most significantly over-represented disease, with an enrichment ratio of 78 (PULMONARY FIBROSIS, IDIOPATHIC, gene set 178500, FDR= 0.00025). 
Cdc42 is an established target of miR-608 [9]. Moreover, it was recently implicated in lung fibrosis, as it was found that the loss of Cdc42 promotes the fibrotic process [11]. Thus, we measured the expression level of both Cdc42 mRNA variants (NM_001791 and NM_044472) in IPF patient samples, in comparison to controls. Both Cdc42 isotype levels in IPF patient samples were significantly lower compared with those of the control samples ( $p<0.001$ and $p<0.001$, respectively, Figure $2 A-B$ ).

\section{IL-6 levels are downregulated in IPF patient samples}

IL-6 is well-known cytokine in IPF and a validated target of miR-608. In our recent work, we showed that the IL-6R protein level is reduced in tissue samples taken from IPF patient biopsies [12]. qPCR was performed to measure the expression of IL- 6 in IPF patients compared with healthy individuals. In fact, IL6 levels were lower in the IPF patient FFPE samples compared with controls $(p<0.05$, Figure $2 C)$. This result is in key with the hypothesis stating an inhibitory relationship between miRNA-608 and its targets.

\section{The C2098A substitution (minor rs17228616 allele) at the AChE sequence is more prevalent in IPF patients}

It was previously shown that the miR-608 has differential affinity to the AchE sequence due to $C$ to $A$ change (C2098A) in its 3'-untranslated region (e.g. the minor 'A-allele' and 'C-allele', respectively), indicating weakened A-allele AChE-miR-608 interaction [9]. The impaired interaction of the A-allele AChE with miR-608 predicted both weakened AChE suppression and freed more miR-608 molecules to suppress other targets with tighter binding parameters, such as Cdc42 and IL6, as found in our results.

We hypothesized that the presence of the minor A-allele could be higher in subjects with IPF. Thus, 56 patients were recruited and their DNA was sequenced for the $A / C$ allele. Patient characteristics are listed in Table 2. $62.5 \%$ were male with the average age of $65.82 \pm 12$.

Of these subjects, the frequency of the A-allele was $17 / 56$ (30.4\%) with all patients being heterozygous for the minor A-allele (Figure 3 ). This result is significant vs. the published Israeli cohort of healthy individuals, which reported $17 \%$ prevalence of this allele in healthy control volunteers $(p=0.01, O R=2.1, \mathrm{Cl}$ 95\% [1.19-3.9]).

Of them, $64.3 \%$ were diagnosed with IPF and the rest with other types of ILD (e.g. NSIP, silicosis etc.). In addition, we followed up these patients' disease progression to determine whether they are rapidly progressing, as previously defined by the annual decline in FVC\% [13-16]. We found that of the minor allele population, more patients presented with a rapid progressing disease $(35.4 \%$ vs. $20 \%, p=0.24)$, yet this result did not reach significance.

When comparing between the $A$ to the $C$ allele groups' lung function tests at the time of diagnosis, $a$ significantly reduced FVC at diagnosis for the A group was observed (Table 2, $p=0.02$ ). 
Table 2:

Patient characteristics

\begin{tabular}{|llll|}
\hline Parameter & A-allele & C-allele & p-value \\
& $\mathbf{n = 1 7}$ & $\mathbf{n = 3 9}$ & \\
\hline Age & $62 \pm 14$ & $62.4 \pm 11$ & 0.9 \\
\hline Gender (\%male) & $12(70 \%)$ & $23(58.9 \%)$ & 0.41 \\
\hline Smoker & $13(76.4 \%)$ & $20(51.2 \%)$ & 0.08 \\
\hline Rapidly progressing disease & $6(35.2 \%)$ & $8(20.5 \%)$ & 0.24 \\
\hline FVC \% & $59 \pm 14.5$ & $70 \pm 19$ & 0.05 \\
\hline DLCO \% & $46.2 \pm 17$ & $48.6 \pm 17$ & 0.66 \\
\hline BMI & $27.6 \pm 5$ & $28.4 \pm 4.5$ & 0.61 \\
\hline IHD & $5(29.4 \%)$ & $7(17.9 \%)$ & 0.34 \\
\hline CHF & $1(5.9 \%)$ & $2(5.1 \%)$ & 0.9 \\
\hline Diabetes & $7(41.2 \%)$ & $16(41 \%)$ & 1 \\
\hline Anxiety & $1(5.9 \%)$ & $6(15.4 \%)$ & 0.3 \\
\hline Hypertension & $6(35.2 \%)$ & $11(28.2 \%)$ & 0.64 \\
\hline Osteoporosis & $4(23.5 \%)$ & $7(17.9 \%)$ & 0.63 \\
\hline Malignancy & $1(5.9 \%)$ & $5(12.8 \%)$ & 0.44 \\
\hline
\end{tabular}

\section{Discussion}

Numerous pathways were implicated and major progress has been made towards understanding IPF etiology [17]. However, as recently stated by McDonough et el, since the UIP pattern cannot be replicated in animal models, we have no information about what regulates progression of IPF in the human lung [18]. Thus, in this work we focused on the primate specific miRNA that was previously implicated in aging related diseases. We found that miR-608 was significantly upregulated in IPF tissue samples. In addition, we found that the minor rs 17228616 allele was more abundant in IPF patients than in the general population.

miR-608 is located on human chromosome 10q24.31. Although not afermentioned in IPF [19], current studies in tumors indicate that miR-608 affects cell proliferation, invasion, migration and apoptosis [20, 21]. Although the expression level of miR-608 was found to be downregulated in several types of cancer $[8,22-25]$, these studies did not take into account the SNPs that can significantly affect miRNA stability and function [7]. 
The major SNP of miR-608 mentioned in cancer is the rs4919510 variant $\mathrm{G}$ allele. This SNP was suggested to affect the expression of mature miR-608, as well as that of the proinflammatory cytokines TNF- $a$, IL- 6 , and IL-1 $\beta$. Nevertheless, there are conflicting results for the association between the presence of miR-608 rs4919510 and susceptibility to tumors. [8, 26-28]. Although IPF and lung cancer are sometimes seen in the same patients [29], since we observed an upregulation in the miR-608, rs4919510 was not studied and our focus was shifted to other directions.

To date, limited targets of miR-608 have been confirmed by in-vitro studies performed in mostly tumor cell lines [30]. A recent study by Wu et al, suggested that Cdc42 is an important post-transcriptional regulator and may play a significant role in the process of inflammation. Although IPF is not considered to be an inflammatory disease per se, pro-inflammatory factors, such as IL-6, TNF-alpha and IFN-y were shown to contribute to disease progression $[12,31]$.

Several studies reported that symptoms of depression and anxiety are common in patients with IPF. Such studies indicated prevalence of depression ranges from $24.3-49.2 \%$, while that of anxiety reaching $60 \%$ in patients with IPF and other ILDs [32-36]. Although a causality of anxiety or depression could be expected, it is possible to assume a genetic predisposition as well.

Our work was inspired by a group of researchers who studied miR-608 in the context of anxiety [9]. They investigated the interaction of miR-608 with AChE (major rs17228616) and the resulting changes, which give rise to a higher ratio of suppression by miR-608 of its other targets, including CdC42 and IL-6, as shown in our results. They showed that young, healthy volunteers with the minor rs17228616 allele showed elevated blood pressure and reduced cortisol, predicting risk of aging-related diseases, such as IPF. Our cohort of patients with fibrosing ILDs, mostly IPF, was shown to include significantly higher prevalence of minor A-allele in comparison to the healthy cohort presented by this group. Since both populations were from Israel, we can also assume similar genetic backgrounds. These results require further investigation in a large cohort to determine the polymorphism in this patient population.

In conclusion, although the number of patients was limited, a significant effect was reached. We found that miR-608 is overexpressed in IPF patients and that this patient population includes 30 percent of a specific SNP in AChE that was previously implicated as relevant to aging related diseases. These findings require further research in a large study cohort.

\section{Declarations}

\section{Ethics approval and consent to participate:}

The study was approved by the Ethics Committee of Meir Medical Center. Signed informed consent was obtained from all patients.

\section{Consent for publication:}

Not applicable 
Availability of data and material:

Any data can be supplemented on demand.

\section{Competing interests:}

all authors declare no conflict of interest and have consented for publication.

\section{Funding:}

no funding was received for this work

\section{Acknowledgements:}

The authors thank Ms. Tatiana Epstein for the English editing.

\section{Authors' contributions:}

GES drafted the manuscript, designed the experiments and analyzed the results, IK performed the experiments and analyzed the results, OW revised it critically for important intellectual content, while DS and LIS contributed to conception and design, drafting the manuscript for important intellectual content and revised the final version.

\section{References}

1. Oldham JM, Collard HR Comorbid Conditions in Idiopathic Pulmonary Fibrosis: Recognition and Management. Front Med (Lausanne) 2017, 4:123.

2. Raghu G, Collard HR, Egan JJ, Martinez FJ, Behr J, Brown KK, Colby TV, Cordier JF, Flaherty KR, Lasky JA, et al An official ATS/ERS/JRS/ALAT statement: idiopathic pulmonary fibrosis: evidence-based guidelines for diagnosis and management. Am J Respir Crit Care Med 2011, 183:788-824.

3. Tashiro J, Rubio GA, Limper AH, Williams K, Elliot SJ, Ninou I, Aidinis V, Tzouvelekis A, Glassberg MK Exploring Animal Models That Resemble Idiopathic Pulmonary Fibrosis. Front Med (Lausanne) 2017, $4: 118$.

4. Noth I, Zhang Y, Ma SF, Flores C, Barber M, Huang Y, Broderick SM, Wade MS, Hysi P, Scuirba J, et al Genetic variants associated with idiopathic pulmonary fibrosis susceptibility and mortality: a genome-wide association study. Lancet Respir Med 2013, 1:309-317.

5. Manichaikul A, Wang XQ, Sun L, Dupuis J, Borczuk AC, Nguyen JN, Raghu G, Hoffman EA, OnengutGumuscu S, Farber EA, et al Genome-wide association study of subclinical interstitial lung disease in MESA. Respir Res 2017, 18:97.

6. Ahn MH, Park BL, Lee SH, Park SW, Park JS, Kim DJ, Jang AS, Park JS, Shin HK, Uh ST, et al A promoter SNP rs4073T>A in the common allele of the interleukin 8 gene is associated with the 
development of idiopathic pulmonary fibrosis via the IL-8 protein enhancing mode. Respir Res 2011, $12: 73$.

7. Duan R, Pak C, Jin P Single nucleotide polymorphism associated with mature miR-125a alters the processing of pri-miRNA. Hum Mol Genet 2007, 16:1124-1131.

8. Wang YF, Ao X, Liu Y, Ding D, Jiao WJ, Yu Z, Zhai WX, Dong SH, He YQ, Guo H, Wang JX MicroRNA608 Promotes Apoptosis in Non-Small Cell Lung Cancer Cells Treated With Doxorubicin Through the Inhibition of TFAP4. Front Genet 2019, 10:809.

9. Hanin G, Shenhar-Tsarfaty S, Yayon N, Yau YH, Bennett ER, Sklan EH, Rao DC, Rankinen T, Bouchard C, Geifman-Shochat S, et al Competing targets of microRNA-608 affect anxiety and hypertension. Hum Mol Genet 2014, 23:4569-4580.

10. Kang JG, Majerciak V, Uldrick TS, Wang X, Kruhlak M, Yarchoan R, Zheng ZM Kaposi's sarcomaassociated herpesviral IL- 6 and human IL- 6 open reading frames contain miRNA binding sites and are subject to cellular miRNA regulation. J Pathol 2011, 225:378-389.

11. Wu H, Yu Y, Huang H, Hu Y, Fu S, Wang Z, Shi M, Zhao X, Yuan J, Li J, et al Progressive Pulmonary Fibrosis Is Caused by Elevated Mechanical Tension on Alveolar Stem Cells. Cell 2020, 180:107-121 e117.

12. Epstein Shochet G, Brook E, Bardenstein-Wald B, Shitrit D TGF-beta pathway activation by idiopathic pulmonary fibrosis (IPF) fibroblast derived soluble factors is mediated by IL- 6 trans-signaling. Respir Res 2020, 21:56.

13. McCormack FX, King TE, Jr., Bucher BL, Nielsen L, Mason RJ Surfactant protein A predicts survival in idiopathic pulmonary fibrosis. Am J Respir Crit Care Med 1995, 152:751-759.

14. Selman M, Carrillo G, Estrada A, Mejia M, Becerril C, Cisneros J, Gaxiola M, Perez-Padilla R, Navarro C, Richards T, et al Accelerated variant of idiopathic pulmonary fibrosis: clinical behavior and gene expression pattern. PLoS One 2007, 2:e482.

15. Trujillo G, Meneghin A, Flaherty KR, Sholl LM, Myers JL, Kazerooni EA, Gross BH, Oak SR, Coelho AL, Evanoff $\mathrm{H}$, et al TLR9 differentiates rapidly from slowly progressing forms of idiopathic pulmonary fibrosis. Sci Transl Med 2010, 2:57ra82.

16. Balestro E, Calabrese F, Turato G, Lunardi F, Bazzan E, Marulli G, Biondini D, Rossi E, Sanduzzi A, Rea $F$, et al Immune Inflammation and Disease Progression in Idiopathic Pulmonary Fibrosis. PLoS One 2016, 11:e0154516.

17. Lederer DJ, Martinez FJ Idiopathic Pulmonary Fibrosis. N Engl J Med 2018, 379:797-798.

18. McDonough JE, Ahangari F, Li Q, Jain S, Verleden SE, Herazo-Maya J, Vukmirovic M, Deluliis G, Tzouvelekis A, Tanabe N, et al Transcriptional regulatory model of fibrosis progression in the human lung. JCl Insight 2019, 4.

19. Bagnato G, Roberts WN, Roman J, Gangemi S A systematic review of overlapping microRNA patterns in systemic sclerosis and idiopathic pulmonary fibrosis. Eur Respir Rev 2017, 26.

20. Wang Z, Xue Y, Wang P, Zhu J, Ma J MiR-608 inhibits the migration and invasion of glioma stem cells by targeting macrophage migration inhibitory factor. Oncol Rep 2016, 35:2733-2742. 
21. Liang Z, Wang X, Xu X, Xie B, Ji A, Meng S, Li S, Zhu Y, Wu J, Hu Z, et al MicroRNA-608 inhibits proliferation of bladder cancer via AKT/FOXO3a signaling pathway. Mol Cancer 2017, 16:96.

22. Miao Z, Guo X, Tian $L$ The long noncoding RNA NORAD promotes the growth of gastric cancer cells by sponging miR-608. Gene 2019, 687:116-124.

23. Zhang Y, Schiff D, Park D, Abounader R MicroRNA-608 and microRNA-34a regulate chordoma malignancy by targeting EGFR, Bcl-xL and MET. PLoS One 2014, 9:e91546.

24. Yang PW, Huang YC, Hsieh CY, Hua KT, Huang YT, Chiang TH, Chen JS, Huang PM, Hsu HH, Kuo SW, et al Association of miRNA-related genetic polymorphisms and prognosis in patients with esophageal squamous cell carcinoma. Ann Surg Oncol 2014, 21 Suppl 4:S601-609.

25. Yu HX, Wang XM, Han XD, Cao BF MiR-608 exerts tumor suppressive function in lung adenocarcinoma by directly targeting MIF. Eur Rev Med Pharmacol Sci 2018, 22:4908-4916.

26. Dai ZM, Kang HF, Zhang WG, Li HB, Zhang SQ, Ma XB, Lin S, Wang M, Feng YJ, Liu K, et al The Associations of Single Nucleotide Polymorphisms in miR196a2, miR-499, and miR-608 With Breast Cancer Susceptibility: A STROBE-Compliant Observational Study. Medicine (Baltimore) 2016, 95:e2826.

27. Pardini B, Rosa F, Naccarati A, Vymetalkova V, Ye Y, Wu X, di Gaetano C, Buchler T, Novotny J, Matullo $G$, Vodicka P Polymorphisms in microRNA genes as predictors of clinical outcomes in colorectal cancer patients. Carcinogenesis 2015, 36:82-86.

28. Li MP, Hu YD, Hu XL, Zhang YJ, Yang YL, Jiang C, Tang J, Chen XP MiRNAs and miRNA Polymorphisms Modify Drug Response. Int J Environ Res Public Health 2016, 13.

29. Gershman E, Zer A, Pertzov B, Shtraichman O, Shitenberg D, Heching M, Rosengarten D, Kramer M Characteristics of lung cancer in idiopathic pulmonary fibrosis with single lung transplant versus non-transplanted patients: a retrospective observational study. BMJ Open Respir Res 2020, 7.

30. Gu W, Wen D, Lu H, Zhang A, Wang H, Du J, Zeng L, Jiang J MiR-608 Exerts Anti-inflammatory Effects by Targeting ELANE in Monocytes. J Clin Immunol 2019.

31. Le TT, Karmouty-Quintana H, Melicoff E, Le TT, Weng T, Chen NY, Pedroza M, Zhou Y, Davies J, Philip K, et al Blockade of IL-6 Trans signaling attenuates pulmonary fibrosis. J Immunol 2014, 193:37553768.

32. Lee YJ, Choi SM, Lee YJ, Cho YJ, Yoon HI, Lee JH, Lee CT, Park JS Clinical impact of depression and anxiety in patients with idiopathic pulmonary fibrosis. PLoS One 2017, 12:e0184300.

33. Akhtar AA, Ali MA, Smith RP Depression in patients with idiopathic pulmonary fibrosis. Chron Respir Dis 2013, 10:127-133.

34. Ryerson CJ, Berkeley J, Carrieri-KohIman VL, Pantilat SZ, Landefeld CS, Collard HR Depression and functional status are strongly associated with dyspnea in interstitial lung disease. Chest 2011, 139:609-616.

35. Lindell KO, Olshansky E, Song MK, Zullo TG, Gibson KF, Kaminski N, Hoffman LA Impact of a disease-management program on symptom burden and health-related quality of life in patients with idiopathic pulmonary fibrosis and their care partners. Heart Lung 2010, 39:304-313. 
36. Holland AE, Fiore JF, Jr., Bell EC, Goh N, Westall G, Symons K, Dowman L, Glaspole I Dyspnoea and comorbidity contribute to anxiety and depression in interstitial lung disease. Respirology 2014, 19:1215-1221.

\section{Figures}

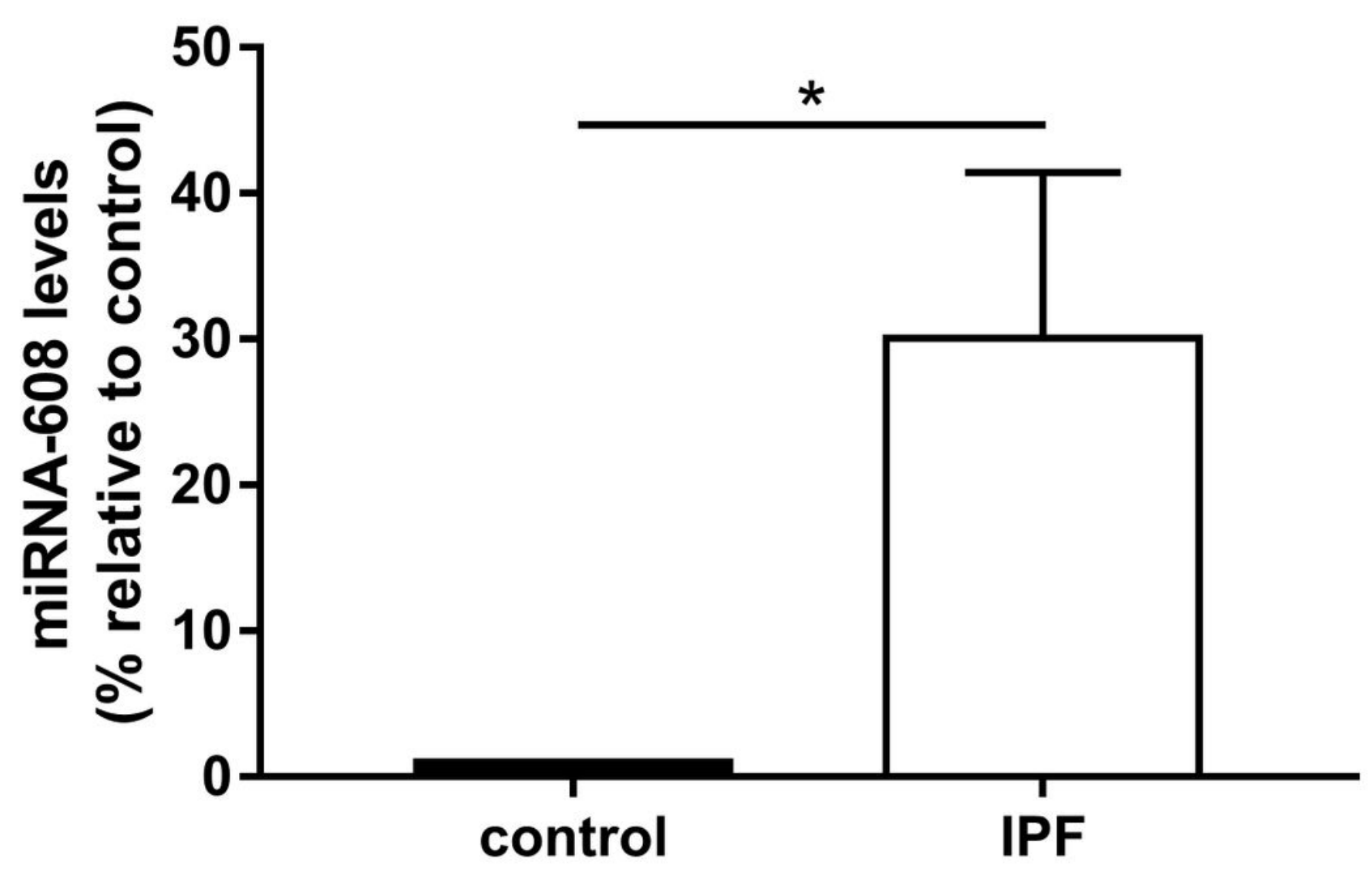

Figure 1

miR-608 is overexpressed in lung tissue samples from IPF patients RNA was extracted from IPF and nonIPF (control) FFPE samples. miRNA-608 levels were evaluated by qPCR. ${ }^{\star} * \star p \leq 0.001$, Student's paired ttest $(n>3)$.
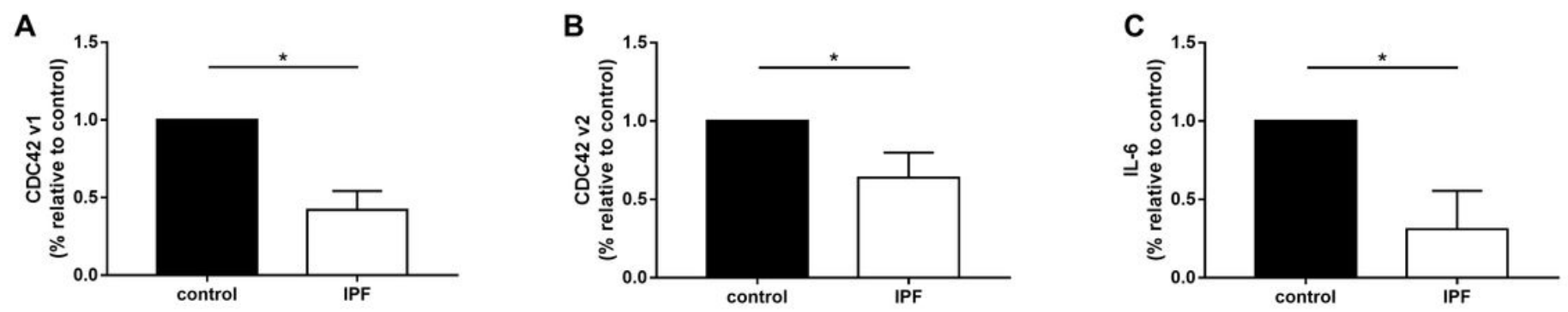
Figure 2

miR-608 target levels are significantly reduced in IPF patient samples RNA was extracted from IPF and non-IPF (control) FFPE samples. The two variants of Cdc42 (A-B) and IL-6 (C) mRNA levels were evaluated by $q P C R .{ }^{*} p \leq 0.5$, Student's paired t-test $(n>3)$.
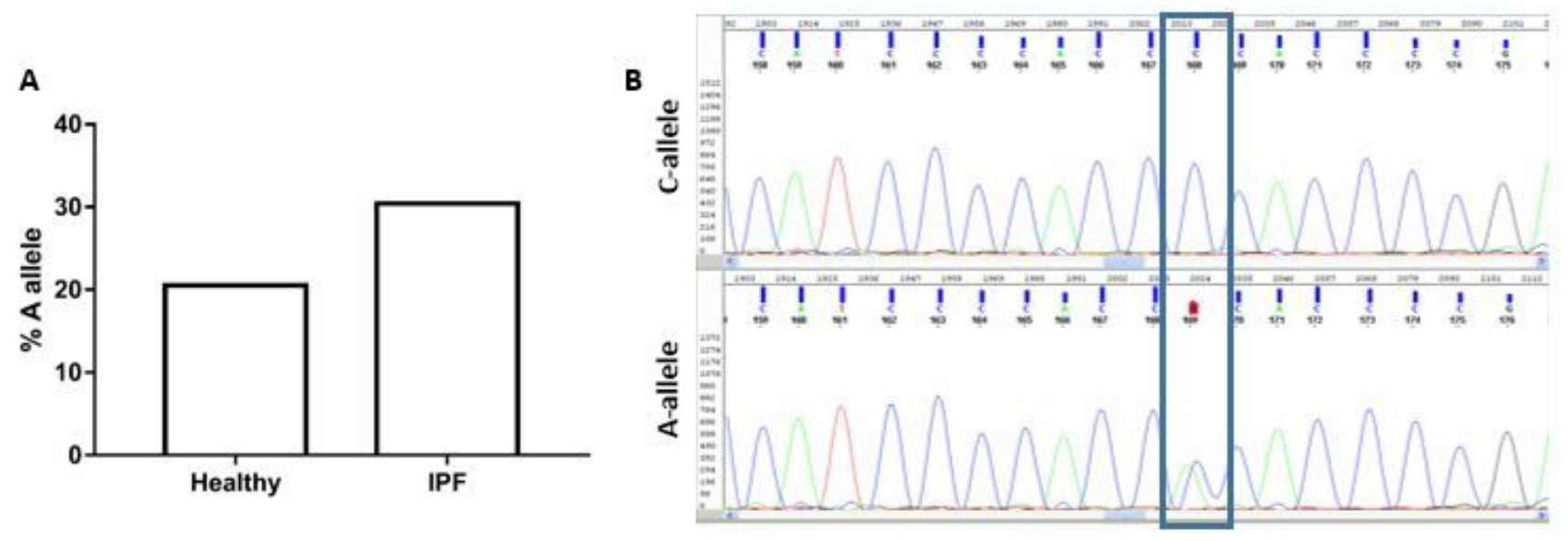

\section{Figure 3}

The C2098A substitution (minor rs17228616 allele) at the AChE sequence is more prevalent in IPF patients DNA was extracted from 56 subjects with progressive fibrosing ILD. AChE miR-609 target sequence was analysed for determining A/C allele rs17228616 SNP (A). $B$ is a representative image of sequencing output showing $C$ (top panel) and $A$ heterozygous allele (bottom panel). 